\title{
Well-Posedness to the Cauchy Problem of a Fully Dispersive Boussinesq System
}

\section{Yuexun Wang ${ }^{1}$}

Received: 7 August 2019 / Revised: 28 January 2020 / Published online: 20 February 2020

(c) The Author(s) 2020

\begin{abstract}
This work concerns the local well-posedness to the Cauchy problem of a fully dispersive Boussinesq system which models fully dispersive water waves in two and three spatial dimensions. Our purpose is to understand the modified energy approach (Kalisch and Pilod in Proc Am Math Soc 147:2545-2559, 2019) in a different point view by utilizing the symmetrization of hyperbolic systems which produces an equivalent modified energy.
\end{abstract}

Keywords Well-posedness · Dispersive Boussinesq system · Symmetrization

Mathematics Subject Classification 76B15 · 76B03 $\cdot 35 \mathrm{~S} 30$

\section{Introduction and Main Results}

The one-dimensional fully dispersive Boussinesq system is governed by

$$
\left\{\begin{array}{l}
\partial_{t} \eta=-\mathcal{K} \partial_{x} u-\partial_{x}(\eta u), \\
\partial_{t} u=-\partial_{x} \eta-u \partial_{x} u,
\end{array}\right.
$$

here $t, x \in \mathbb{R}$, and the surface elevation $\eta$ and the velocity $u$ at the surface are real functions. We also consider the two-dimensional fully dispersive Boussinesq system which reads as:

$$
\left\{\begin{array}{l}
\partial_{t} \eta=-\mathcal{K} \operatorname{div} \boldsymbol{v}-\operatorname{div}(\eta \boldsymbol{v}), \\
\partial_{t} \boldsymbol{v}=-\nabla \eta-\frac{1}{2} \nabla|\boldsymbol{v}|^{2},
\end{array}\right.
$$

in which $t \in \mathbb{R}, x=\left(x_{1}, x_{2}\right) \in \mathbb{R}^{2}$ and the unknowns $\eta \in \mathbb{R}, \boldsymbol{v}=\left(v_{1}, v_{2}\right) \in \mathbb{R}^{2}$. The operator $\mathcal{K}$ is a Fourier multiplier with symbol $m \in S_{\infty}^{a}\left(\mathbb{R}^{d}\right), d=1$ or 2 for some $a \in \mathbb{R} \backslash\{0\}$,

Y.W. acknowledges the support by Grants Nos. 231668 and 250070 from the Research Council of Norway.

\footnotetext{
$\bowtie$ Yuexun Wang

yuexunwang@1zu.edu.cn

1 School of Mathematics and Statistics, Lanzhou University, 370000 Lanzhou, China
} 
that is

$$
\mathcal{F}(\mathcal{K} f)(\xi)=m(\xi) \hat{f}(\xi), \quad \forall f \in \mathcal{S}\left(\mathbb{R}^{d}\right),
$$

for some smooth even function $m: \mathbb{R}^{d} \rightarrow \mathbb{R}^{d}$ with the properties

$$
m(\xi) \approx(1+|\xi|)^{a},
$$

and

$$
\left|m^{\prime}(\xi)\right| \lesssim(1+|\xi|)^{a-1} .
$$

The system (1.1) was formally derived in $[1,16]$ from the incompressible Euler equations to model fully dispersive water waves whose propagation is allowed to be both left- and rightward, and was also proposed in $[13,14,19]$ (as well as the system (1.2)) as a full dispersion system in the Boussinesq regime with the dispersion of the water waves system. This model is the two-way equivalent of the one-dimensional Whitham equation

$$
\partial_{t} u=-\mathcal{K}^{1 / 2} \partial_{x} u-u \partial_{x} u,
$$

with $m(\xi)=\tanh (\xi) / \xi$, which has taken a vast of attractions (we refer to [7] for a fairly complete list of references). There have been several investigations on the system (1.1) with $m(\xi)=\tanh (\xi) / \xi$ [which corresponds to $a=-1$ in (1.3)]: local well-posedness [13,18], a logarithmically cusped wave of greatest height [6], existence of solitary wave solutions [17], and numerical results $[3,4,21]$. We should point out that the assumption in $[13,18]$ on the initial surface elevation $\eta_{0} \geq C>0$ is nonphysical, which only yields the well-posedness in homogeneous Sobolev spaces, however the Cauchy problem of (1.1) by this choice of symbol is probably ill-posed for negative initial surface elevation (see a heuristic argument in [13]).

When $a>0$ in (1.3), because of the strong dispersion, the system (1.1) exhibits different phenomenon regarding well-posedness. Very recently, considering the effect of surface tension, i.e., $m(\xi)=(\tanh (|\xi|) /|\xi|)\left(1+|\xi|^{2}\right)$ [which corresponds to $a=1$ in (1.3)], the well-posedness in Sobolev spaces was shown in [10] for the Cauchy problem of the system (1.1), as well as (1.2) with curl free initial velocity $\boldsymbol{v}_{0}$. Due to the lack of symmetry of the nonlinearity, the usual energy estimates only give

$$
\begin{aligned}
\frac{\mathrm{d}}{\mathrm{d} t}\left(\|\eta\|_{H^{s}}^{2}+\|u\|_{H^{s+\frac{1}{2}}}^{2}\right) \lesssim & \left(1+\|\eta\|_{H^{s}}+\|u\|_{H^{s+\frac{1}{2}}}\right)\left(\|\eta\|_{H^{s}}^{2}+\|u\|_{H^{s+\frac{1}{2}}}^{2}\right) \\
& +\left|\int \eta J^{s} \partial_{x} u J^{s} \eta \mathrm{d} x\right| .
\end{aligned}
$$

To close the resulting energy estimate, the crucial idea is to define a modified energy, i.e., adding a lower order cubic term $\int \eta\left(J^{s} \partial_{x} u\right)^{2} \mathrm{~d} x$ (which has no fixed sign) to the LHS of (1.5). Another important observation is that the non-cavitation assumption on $\eta$ (which means that the surface elevation of the wave cannot touch the bottom of the fluid) allows $\|u\|_{H^{s+\frac{1}{2}}}^{2}$ to control the part $\|u\|_{H^{s+\frac{1}{2}}}^{2}+\int \eta\left(J^{s} \partial_{x} u\right)^{2} \mathrm{~d} x$ of the modified energy from below. Besides, the authors also used quite a few tricks such as some operator decompositions (to reformulate the system (1.1)), and the estimate comparing the Bessel and Riesz potentials, and so on. Please refer to $[5,8,9,12]$ for other applications of modified energy approaches in some related contexts of dispersive equations. We also refer to $[13,15]$ for discussions and interesting issues about the influence of dispersion on the lifespan of solutions to dispersive perturbations of hyperbolic quasi-linear equations or systems which typically arise in water waves theory. 
Inspired by [10,13], our purpose is to understand the modified energy approach in a different point view. Our idea is to use symmetrization of hyperbolic systems, which will help us to find the modified energy easily. The energy estimates are straightforward and yield

$$
\begin{aligned}
& \frac{\mathrm{d}}{\mathrm{d} t} \int\left(\left(J^{s} \eta\right)^{2}+\eta\left(J^{s} u\right)^{2}+\left(\sqrt{\mathcal{K}} J^{s} u\right)^{2}\right) \mathrm{d} x \\
& \quad \lesssim\left(\|\eta\|_{H^{s}}+\|u\|_{H^{s}}+\|\eta\|_{H^{s}}\|u\|_{H^{s}}\right)^{3}+\|u\|_{H^{s}}\|u\|_{H^{s+\frac{a}{2}}}^{2}
\end{aligned}
$$

The integration in the LHS of (1.6) is exactly the modified energy that we need. Notice that

$$
\int\left(\sqrt{\mathcal{K}} J^{s} u\right)^{2} \mathrm{~d} x \approx\|u\|_{H^{s+\frac{a}{2}}}^{2},
$$

so we see that this modified energy is essentially same to the one in [10]. On the other hand, this zeroth-order $[s=0]$ modified energy is corresponding to the Hamiltonian of the system (1.1) (up to a constant coefficient). We also refer to [20] for using the idea of symmetrization to study dispersive systems.

We start from recalling the definition of the non-cavitation:

Definition 1.1 ([10]) Let $d=1$ or 2 and $k>\frac{d}{2}$. We say that the initial elevation $\eta_{0} \in H^{k}\left(\mathbb{R}^{d}\right)$ satisfies the non-cavitation condition if there exists $h_{0} \in(0,1)$ such that

$$
1+\eta_{0}(x) \geq h_{0}, \quad x \in \mathbb{R}^{d} .
$$

We then have similar results to [10] under the non-cavitation assumption:

Theorem 1.2 Let $a>0$ and $s>\frac{3}{2}+a$. Assume that $\left(\eta_{0}, u_{0}\right) \in H^{s}(\mathbb{R}) \times H^{s+\frac{a}{2}}(\mathbb{R})$ satisfy the non-cavitation condition (1.7). Then there exist a positive number

$$
T=T\left(\left\|\left(\eta_{0}, u_{0}\right)\right\|_{H^{s}(\mathbb{R}) \times H^{s+\frac{a}{2}}(\mathbb{R})}\right)
$$

and a unique solution

$$
(\eta, u) \in C\left([0, T] ; H^{s}(\mathbb{R}) \times H^{s+\frac{a}{2}}(\mathbb{R})\right)
$$

to $(1.1)$ with $(\eta, u)(0, x)=\left(\eta_{0}, u_{0}\right)(x)$. In addition, the flow function that maps initial data to solutions is continuous.

Theorem 1.3 Let $a>0$ and $s>2+a$. Assume that $\left(\eta_{0}, \boldsymbol{v}_{0}\right) \in H^{s}\left(\mathbb{R}^{2}\right) \times H^{s+\frac{a}{2}}\left(\mathbb{R}^{2}\right)^{2}$ satisfy the non-cavitation condition (1.7) and $\operatorname{curl} v_{0}=0$. Then there exist a positive number

$$
T=T\left(\left\|\left(\eta_{0}, \boldsymbol{v}_{0}\right)\right\|_{H^{s}\left(\mathbb{R}^{2}\right) \times H^{s+\frac{a}{2}}\left(\mathbb{R}^{2}\right)^{2}}\right)
$$

and a unique solution

$$
(\eta, \boldsymbol{v}) \in C\left([0, T] ; H^{s}\left(\mathbb{R}^{2}\right) \times H^{s+\frac{a}{2}}\left(\mathbb{R}^{2}\right)^{2}\right)
$$

to $(1.2)$ with $(\eta, v)(0, x)=\left(\eta_{0}, v_{0}\right)(x)$. In addition, the flow function that maps initial data to solutions is continuous.

To end this section, we include some notations frequently used throughout this paper. Let $\langle x\rangle^{l}=\left(1+|x|^{2}\right)^{\frac{l}{2}}$ and $\widehat{J^{l} f}(\xi)=\langle\xi\rangle^{l} \hat{f}(\xi)$, and denote by $H^{l}$ the $L^{2}$ based Sobolev space with the norm $\|\cdot\|_{H^{l}}=\left\|J^{l} \cdot\right\|_{L^{2}}$. The notation $C$ always denotes a nonnegative universal constant which may be different from line to line but is independent of the parameters involved. Otherwise, we will specify it by the notation $C(a, b, \ldots)$. We write $f \lesssim g[f \gtrsim g]$ when $f \leq C g[f \geq C g]$, and $f \approx g$ when $f \lesssim g \lesssim f$. 


\section{Proof of Theorem 1.2}

\subsection{Energy Estimates}

Denote

$$
E(\eta, u)(t)=\int\left(\left(J^{s} \eta\right)^{2}+\eta\left(J^{s} u\right)^{2}+\left(\sqrt{\mathcal{K}} J^{s} u\right)^{2}\right) \mathrm{d} x
$$

We priorly assume the solution $\eta$ satisfies the non-cavitation assumption: there exists $h_{1} \in$ $(0,1)$ such that

$$
1+\eta(t, x) \geq h_{1}, \quad(x, t) \in \mathbb{R} \times[0, T],
$$

and the upper bound assumption: there exists $h_{2}>0$ such that

$$
\eta(t, x) \leq h_{2}, \quad(x, t) \in \mathbb{R} \times[0, T],
$$

where the time $T$ will be determined by the energy estimate below, and aim to show that there exists some appropriately small positive number $T$ such that

$$
E(\eta, u)(t) \leq C\left(T, a, h_{1}, h_{2}\right) E(\eta, u)(0), \quad \forall t \in[0, T] .
$$

To prove (2.4), we will use the idea of symmetrization of hyperbolic systems, which will help us to find the modified energy $E(\eta, u)(t)$ in (2.1) easily. Let

$$
A=\left[\begin{array}{ll}
1 & 0 \\
0 & \eta
\end{array}\right], B=\left[\begin{array}{cc}
u & \eta \\
\eta & \eta u
\end{array}\right], U=\left[\begin{array}{l}
\eta \\
u
\end{array}\right], G=\left[\begin{array}{c}
\mathcal{K} \partial_{x} u \\
0
\end{array}\right]
$$

Multiplying (1.1) by the matrix $A$ yields the following system

$$
A \partial_{t} U+B \partial_{x} U+G=0 .
$$

Applying $J^{S}$ to (2.5) and multiplying it by $J^{S} U$ give

$$
\begin{aligned}
\frac{1}{2} \frac{\mathrm{d}}{\mathrm{d} t} \int J^{s} U \cdot A \cdot J^{s} U \mathrm{~d} x \\
=\underbrace{\frac{1}{2} \int J^{s} U \cdot \partial_{t} A \cdot J^{s} U \mathrm{~d} x}_{I_{1}}-\underbrace{\int J^{s} U \cdot\left[J^{s}\left(A \partial_{t} U\right)-A J^{s} \partial_{t} U\right] \mathrm{d} x}_{I_{3}} \\
-\underbrace{\int J^{s} U \cdot\left[J^{s}\left(B \partial_{x} U\right)-B J^{s} \partial_{x} U\right] \mathrm{d} x}_{I_{2}}-\underbrace{\int J^{s} U \cdot B \cdot \partial_{x} J^{s} U \mathrm{~d} x}_{I_{4}} \\
-\underbrace{\int J^{s} U \cdot J^{s} G \mathrm{~d} x}_{I_{5}} .
\end{aligned}
$$

The terms $I_{2}, I_{3}$ and $I_{4}$ come from the hyperbolic parts [do not involve the Fourier multiplier $\mathcal{K}$ ], and therefore can be handled either by Kato-Ponce's commutator estimates [11] or by integration by parts to obtain

$$
\left|I_{2}\right|+\left|I_{3}\right|+\left|I_{4}\right| \lesssim\left(\|\eta\|_{H^{s}}+\|u\|_{H^{s}}+\|\eta\|_{H^{s}}\|u\|_{H^{s}}\right)^{3} .
$$


It remains to treat the terms $I_{1}$ and $I_{5}$. In view of $(1.1)_{1}$, we estimate

$$
\begin{aligned}
\left|I_{1}\right| & =\frac{1}{2}\left|\int\left[\mathcal{K} \partial_{x} u+\partial_{x}(\eta u)\right]\left(J^{s} u\right)^{2} \mathrm{~d} x\right| \lesssim\left\|\mathcal{K} \partial_{x} u+\partial_{x}(\eta u)\right\|_{L^{\infty}}\|u\|_{H^{s}}^{2} \\
& \lesssim\left(\|u\|_{H^{\frac{3}{2}+a+\delta}}+\|\eta\|_{H^{\frac{3}{2}+\delta}}\|u\|_{H^{\frac{3}{2}+\delta}}\right)\|u\|_{H^{s}}^{2} \lesssim\left(1+\|\eta\|_{H^{s}}\right)\|u\|_{H^{s}}^{3},
\end{aligned}
$$

where we have used (1.3), and the embedding $H^{\frac{1}{2}+\delta}(\mathbb{R}) \hookrightarrow L^{\infty}(\mathbb{R})$ with $\delta \in\left(0, s-\frac{3}{2}-a\right)$. Using integration by parts, one calculates

$$
\begin{aligned}
I_{5} & =-\int \sqrt{\mathcal{K}} J^{s} \partial_{x} \eta \cdot \sqrt{\mathcal{K}} J^{s} u \mathrm{~d} x=\int \sqrt{\mathcal{K}} J^{s}\left(\partial_{t} u+u \partial_{x} u\right) \cdot \sqrt{\mathcal{K}} J^{s} u \mathrm{~d} x \\
& =\frac{1}{2} \frac{\mathrm{d}}{\mathrm{d} t} \int\left(\sqrt{\mathcal{K}} J^{s} u\right)^{2} \mathrm{~d} x+\underbrace{\int \sqrt{\mathcal{K}} J^{s}\left(u \partial_{x} u\right) \cdot \sqrt{\mathcal{K}} J^{s} u \mathrm{~d} x}_{I_{6}},
\end{aligned}
$$

where we have inserted the Eq. (1.1 $)_{2}$ into the second equality. To estimate the term $I_{6}$, we commute $u$ out as follows:

$$
I_{6}=\underbrace{\int u \sqrt{\mathcal{K}} J^{s} \partial_{x} u \cdot \sqrt{\mathcal{K}} J^{s} u \mathrm{~d} x}_{I_{7}}+\underbrace{\int\left[\sqrt{\mathcal{K}} J^{s}, u\right] \partial_{x} u \cdot \sqrt{\mathcal{K}} J^{s} u \mathrm{~d} x}_{I_{8}}
$$

The term $I_{7}$, by integration by parts, can be easily controlled by

$$
\left|I_{7}\right|=\frac{1}{2}\left|\int \partial_{x} u\left(\sqrt{\mathcal{K}} J^{s} u\right)^{2} \mathrm{~d} x\right| \lesssim\|u\|_{H^{s}}\|u\|_{H^{s+\frac{a}{2}}}^{2} .
$$

We next estimate the term $I_{8}$. By the Plancherel's theorem, we rewrite

$$
\left\|\left[\sqrt{\mathcal{K}} J^{s}, u\right] \partial_{x} u\right\|_{L^{2}}=\left\|\int\left(\sqrt{m(\xi)}\langle\xi\rangle^{s}-\sqrt{m(\eta)}\langle\eta\rangle^{s}\right) \hat{u}(\xi-\eta) \widehat{\partial_{x} u}(\eta) \mathrm{d} \eta\right\|_{L_{\xi}^{2}} .
$$

Via the mean value theorem, there exists some $\theta \in(0,1)$ such that

$$
\sqrt{m(\xi)}\langle\xi\rangle^{s}-\sqrt{m(\eta)}\langle\eta\rangle^{s}=\left(\frac{m^{\prime}(\zeta)}{2 \sqrt{m(\zeta)}}\langle\zeta\rangle^{s}+s \sqrt{m(\zeta)}\langle\zeta\rangle^{s-2} \zeta\right)(\xi-\eta),
$$

with $\zeta=\theta(\xi-\eta)+(1-\theta) \eta$. Then the above equality and the assumptions (1.3) and (1.4) imply

$$
\begin{aligned}
& \left|\sqrt{m(\xi)}\langle\xi\rangle^{s}-\sqrt{m(\eta)}\langle\eta\rangle^{s}\right| \lesssim\langle\zeta\rangle^{s+\frac{a}{2}-1}|\xi-\eta| \\
& \quad \lesssim\langle\xi-\eta\rangle^{s+\frac{a}{2}-1}|\xi-\eta|+|\xi-\eta|\langle\eta\rangle^{s+\frac{a}{2}-1}
\end{aligned}
$$

Notice that

$$
\begin{aligned}
& \left\|\int|\hat{f}(\xi-\eta) \widehat{g}(\eta)| \mathrm{d} \eta\right\|_{L_{\xi}^{2}} \leq \int\left(\int|\hat{f}(\xi-\eta)|^{2} \mathrm{~d} \xi\right)^{1 / 2}|\widehat{g}(\eta)| \mathrm{d} \eta \\
& \quad \lesssim\|f\|_{L^{2}}\left(\int\left(1+|\eta|^{2}\right)^{b}|\widehat{g}(\eta)|^{2} \mathrm{~d} \eta\right)^{1 / 2}\left(\int\left(1+|\eta|^{2}\right)^{-b} \mathrm{~d} \eta\right)^{1 / 2} \\
& \quad \lesssim\|f\|_{L^{2}}\|g\|_{H^{b}},
\end{aligned}
$$


for any $b>1 / 2$. Substituting (2.12) into (2.11) gives

$$
\left\|\left[\sqrt{\mathcal{K}} J^{s}, u\right] \partial_{x} u\right\|_{L^{2}} \lesssim\left\|\partial_{x} u\right\|_{H^{1}}\left\|J^{s+\frac{a}{2}-1} \partial_{x} u\right\|_{L^{2}},
$$

which leads to

$$
\left|I_{8}\right| \lesssim\|u\|_{H^{s}}\|u\|_{H^{s+\frac{a}{2}}}^{2} .
$$

It follows from (2.6)-(2.10) and (2.13) that

$$
\begin{aligned}
& \frac{\mathrm{d}}{\mathrm{d} t} \int\left(\left(J^{s} \eta\right)^{2}+\eta\left(J^{s} u\right)^{2}+\left(\sqrt{\mathcal{K}} J^{s} u\right)^{2}\right) \mathrm{d} x \\
& \quad \lesssim\left(\|\eta\|_{H^{s}}+\|u\|_{H^{s}}+\|\eta\|_{H^{s}}\|u\|_{H^{s}}\right)^{3}+\|u\|_{H^{s}}\|u\|_{H^{s+\frac{a}{2}}}^{2} .
\end{aligned}
$$

By the assumption (1.3), we have

$$
c^{-1}\|u\|_{H^{s+\frac{a}{2}}}^{2} \leq \int\left(\sqrt{\mathcal{K}} J^{s} u\right)^{2} \mathrm{~d} x \leq c\|u\|_{H^{s+\frac{a}{2}}}^{2} .
$$

We then claim that

$$
E(\eta, u)(t) \bar{\sim}_{a, h_{1}, h_{2}}\|\eta\|_{H^{s}}^{2}+\|u\|_{H^{s+\frac{a}{2}}}^{2}, \quad t \in[0, T] .
$$

The upper bound estimate of (2.16) is an easy consequence of (2.3). We now turn to the lower bound estimate of (2.16). The following observation is due to [10]. By the non-cavitation assumption (2.2), one may estimate

$$
\begin{aligned}
& \int\left(\eta\left(J^{s} u\right)^{2}+\left(\sqrt{\mathcal{K}} J^{s} u\right)^{2}\right) \mathrm{d} x \geq \int\left(\eta\left(J^{s} u\right)^{2}+c^{-1}\left(J^{s+\frac{a}{2}} u\right)^{2}\right) \mathrm{d} x \\
& \geq h_{1} \int\left(1+\xi^{2}\right)^{s}|\hat{u}(\xi)|^{2} \mathrm{~d} \xi+\int\left(c^{-1}\left(1+\xi^{2}\right)^{s+\frac{a}{2}}-\left(1+\xi^{2}\right)^{s}\right)|\hat{u}(\xi)|^{2} \mathrm{~d} \xi \\
& \geq h_{1} C(a) \int_{|\xi| \leq \xi_{0}}\left(1+\xi^{2}\right)^{s+\frac{a}{2}}|\hat{u}(\xi)|^{2} \mathrm{~d} \xi+C(a) \int_{|\xi| \geq \xi_{0}}\left(1+\xi^{2}\right)^{s+\frac{a}{2}}|\hat{u}(\xi)|^{2} \mathrm{~d} \xi \\
& \geq C\left(a, h_{1}\right) \int\left(J^{s+\frac{a}{2}} u\right)^{2} \mathrm{~d} x,
\end{aligned}
$$

where $\xi_{0}>0$ is a number depending on $a$ and $c$. This finishes the proof of (2.16). We then conclude from (2.14)-(2.16) that

$$
E(\eta, u)(t) \lesssim a, h_{1}, h_{2} E(\eta, u)(0)+\int_{0}^{t}\left(E(\eta, u)(\tau)+E^{3}(\eta, u)(\tau)\right) \mathrm{d} \tau,
$$

which together with the Grönwall's inequality completes the proof of (2.4).

\subsection{Estimates for the Differences of Two Solutions}

Assume $i=1,2$. Let $\left(\eta_{i}, u_{i}\right)$ be the solutions of (1.1) with the initial data $\left(\eta_{i_{0}}, u_{i 0}\right)$ and satisfy the non-cavitation assumption (2.2) and the upper bound assumption (2.3). This subsection we instead consider the energy

$$
\tilde{E}(t)=\int\left(\left(\eta_{1}-\eta_{2}\right)^{2}+\eta_{1}\left(u_{1}-u_{2}\right)^{2}+\left(J^{\frac{a}{2}}\left(u_{1}-u_{2}\right)\right)^{2}\right) \mathrm{d} x
$$


and aim to show

$$
\tilde{E}(t) \leq C \tilde{E}(0), \quad \forall t \in[0, T],
$$

where $T$ is defined in (2.4) and $C=C\left(T, a, h_{1}, h_{2}, E\left(\eta_{1}, u_{1}\right)(0), E\left(\eta_{2}, u_{2}\right)(0)\right)$.

We let

$$
A_{i}=\left[\begin{array}{cc}
1 & 0 \\
0 & \eta_{i}
\end{array}\right], B_{i}=\left[\begin{array}{cc}
u_{i} & \eta_{i} \\
\eta_{i} & \eta_{i} u_{i}
\end{array}\right], U_{i}=\left[\begin{array}{l}
\eta_{i} \\
u_{i}
\end{array}\right], G_{i}=\left[\begin{array}{c}
\mathcal{K} \partial_{x} u_{i} \\
0
\end{array}\right],
$$

and take $U=U_{1}$ and $U=U_{2}$ in (2.5), and then find that $\tilde{U}=U_{1}-U_{2}$ solves the following system

$$
A_{1} \partial_{t} \tilde{U}+B_{1} \partial_{x} \tilde{U}+\left(A_{1}-A_{2}\right) \partial_{t} U_{2}+\left(B_{1}-B_{2}\right) \partial_{x} U_{2}+\left(G_{1}-G_{2}\right)=0
$$

Multiplying (2.18) by $\tilde{U}$ yields

$$
\begin{aligned}
& \frac{1}{2} \frac{\mathrm{d}}{\mathrm{d} t} \int \tilde{U} \cdot A_{1} \cdot \tilde{U} \mathrm{~d} x=\underbrace{\frac{1}{2} \int \tilde{U} \cdot \partial_{t} A_{1} \cdot \tilde{U} \mathrm{~d} x}_{I I_{1}}-\underbrace{\int \tilde{U} \cdot B_{1} \cdot \partial_{x} \tilde{U} \mathrm{~d} x}_{I I_{2}} \\
& -\underbrace{\int \tilde{U} \cdot\left(A_{1}-A_{2}\right) \cdot \partial_{t} U_{2} \mathrm{~d} x}_{I I_{3}}-\underbrace{\int \tilde{U} \cdot\left(B_{1}-B_{2}\right) \cdot \partial_{x} U_{2} \mathrm{~d} x}_{I I_{4}} \\
& -\underbrace{\int \tilde{U} \cdot\left(G_{1}-G_{2}\right) \mathrm{d} x}_{I I_{5}} .
\end{aligned}
$$

Since $I I_{2}, I_{3}$ and $I I_{4}$ are usual hyperbolic terms, standard estimates give

$$
\begin{aligned}
& \left|I I_{2}\right|+\left|I I_{3}\right|+\left|I I_{4}\right| \\
& \quad \lesssim\left(\left\|\eta_{1}\right\|_{H^{s}}+\left\|u_{1}\right\|_{H^{s}}+\left\|\eta_{1}\right\|_{H^{s}}\left\|u_{1}\right\|_{H^{s}}+\left\|u_{1}\right\|_{H^{s}}^{2}\right) \\
& \quad \times\left(\left\|\eta_{1}-\eta_{2}\right\|_{L^{2}}^{2}+\left\|u_{1}-u_{2}\right\|_{L^{2}}^{2}+\left\|\eta_{1}-\eta_{2}\right\|_{L^{2}}\left\|u_{1}-u_{2}\right\|_{L^{2}}\right) .
\end{aligned}
$$

In light of the Eqs. (1.1) 1 and the assumption (1.3), we have

$$
\begin{aligned}
\left|I I_{1}\right| & =\frac{1}{2}\left|\int\left[\mathcal{K} \partial_{x} u_{1}+\partial_{x}\left(\eta_{1} u_{1}\right)\right]\left(u_{1}-u_{2}\right)^{2} \mathrm{~d} x\right| \\
& \lesssim\left(\left\|u_{1}\right\|_{H^{s}}+\left\|\eta_{1}\right\|_{H^{s}}\left\|u_{1}\right\|_{H^{s}}\right)\left\|u_{1}-u_{2}\right\|_{L^{2}}^{2} .
\end{aligned}
$$

We now handle the term $I_{5}$ as follows:

$$
\begin{aligned}
I_{5} & =-\int \sqrt{\mathcal{K}} \partial_{x}\left(\eta_{1}-\eta_{2}\right) \cdot \sqrt{\mathcal{K}}\left(u_{1}-u_{2}\right) \mathrm{d} x \\
& =\int \sqrt{\mathcal{K}}\left(\partial_{t}\left(u_{1}-u_{2}\right)+u_{1} \partial_{x} u_{1}-u_{2} \partial_{x} u_{2}\right) \cdot \sqrt{\mathcal{K}}\left(u_{1}-u_{2}\right) \mathrm{d} x \\
& =\frac{1}{2} \frac{\mathrm{d}}{\mathrm{d} t} \int\left(\sqrt{\mathcal{K}}\left(u_{1}-u_{2}\right)\right)^{2} \mathrm{~d} x+\underbrace{\int \sqrt{\mathcal{K}}\left(u_{1} \partial_{x} u_{1}-u_{2} \partial_{x} u_{2}\right) \cdot \sqrt{\mathcal{K}}\left(u_{1}-u_{2}\right) \mathrm{d} x}_{I I_{6}},
\end{aligned}
$$


where we have inserted the Eq. (1.1) $)_{2}$ into the second equality. The term $I I_{6}$ can be dominated by

$$
\left|I I_{6}\right| \lesssim\left(\left\|u_{1}\right\|_{H^{s}}^{2}+\left\|u_{2}\right\|_{H^{s}}^{2}\right)\left\|u_{1}-u_{2}\right\|_{H^{\frac{a}{2}}}
$$

It follows from (2.19)-(2.23) that

$$
\begin{aligned}
& \frac{\mathrm{d}}{\mathrm{d} t} \int\left(\left(\eta_{1}-\eta_{2}\right)^{2}+\eta_{1}\left(u_{1}-u_{2}\right)^{2}+\left(\sqrt{\mathcal{K}}\left(u_{1}-u_{2}\right)\right)^{2}\right) \mathrm{d} x \\
& \leq C\left(E\left(\eta_{1}, u_{1}\right)(t), E\left(\eta_{2}, u_{2}\right)(t)\right)\left(\left\|\eta_{1}-\eta_{2}\right\|_{L^{2}}^{2}+\left\|\eta_{1}-\eta_{2}\right\|_{L^{2}}\left\|u_{1}-u_{2}\right\|_{L^{2}}\right. \\
& \left.\quad+\left\|u_{1}-u_{2}\right\|_{L^{2}}^{2}+\left\|u_{1}-u_{2}\right\|_{H^{\frac{a}{2}}}\right) .
\end{aligned}
$$

Since $\eta_{1}$ satisfies the non-cavitation assumption (2.2), like (2.16), the following equivalence holds:

$$
\tilde{E}(t) \bar{\sim}_{a, h_{1}, h_{2}}\left\|\eta_{1}-\eta_{2}\right\|_{L^{2}}^{2}+\left\|u_{1}-u_{2}\right\|_{H^{\frac{a}{2}}}^{2}, \quad t \in[0, T] .
$$

We then see from (2.24) that

$$
\tilde{E}(t) \lesssim a, h_{1}, h_{2} \tilde{E}(0)+\int_{0}^{t} C\left(E\left(\eta_{1}, u_{1}\right)(\tau), E\left(\eta_{2}, u_{2}\right)(\tau)\right)\left(\tilde{E}(\tau)+\tilde{E}^{1 / 2}(\tau)\right) \mathrm{d} \tau .
$$

This estimate completes the proof of (2.17).

\subsection{Conclusion}

To verify the non-cavitation assumption (2.2), one first rewrites

$$
1+\eta(t, x)=1+\eta_{0}(x)+\int_{0}^{t} \partial_{t} \eta(\tau, x) \mathrm{d} \tau \geq h_{0}-t \sup _{\tau \in[0, t]}\left\|\partial_{t} \eta(\tau, \cdot)\right\|_{L_{x}^{\infty},}
$$

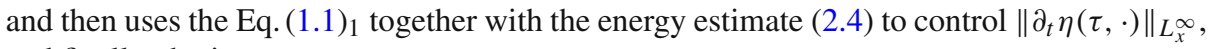
and finally obtains

$$
1+\eta(t, x) \geq h_{0} / 2=: h_{1}, \quad(x, t) \in \mathbb{R} \times[0, T],
$$

by carefully choosing the time $T$. The upper bound assumption (2.3) is a consequence of the energy estimate (2.4) and Sobolev embedding. We refer to [10] for the full details.

Based on the energy estimate (2.4) and the estimate for the differences of two solutions (2.17), it is a standard process following a compactness argument to construct a solution to the Cauchy problem of (1.1) and show the uniqueness of solutions in the solution class stated in Theorem 1.2. Finally one can apply the Bona-Smith argument [2] to verify the continuity of the flow map.

\section{Proof of Theorem 1.3}

\subsection{Energy Estimates}

Taking curl on the equation of $\boldsymbol{v}$ in (1.2) we see that $\operatorname{curl} v=\operatorname{curl} \boldsymbol{v}_{0}=0$, i.e.,

$$
\partial_{x_{1}} v_{2}=\partial_{x_{2}} v_{1} \text {. }
$$


we then use (3.1) to rewrite the system (1.2) into the following form

$$
\left\{\begin{array}{l}
\partial_{t} \eta=-\mathcal{K}\left(\partial_{x_{1}} v_{1}+\partial_{x_{2}} v_{2}\right)-\left(v_{1} \partial_{x_{1}} \eta+v_{2} \partial_{x_{2}} \eta\right)-\eta\left(\partial_{x_{1}} v_{1}+\partial_{x_{2}} v_{2}\right) \\
\partial_{t} v_{1}=-\partial_{x_{1}} \eta-v_{1} \partial_{x_{1}} v_{1}-v_{2} \partial_{x_{2}} v_{1} \\
\partial_{t} v_{2}=-\partial_{x_{2}} \eta-v_{1} \partial_{x_{1}} v_{2}-v_{2} \partial_{x_{2}} v_{2}
\end{array}\right.
$$

Now the system (3.2) allows us to perform the idea of symmetrization as the system (1.1). Let

$$
\begin{aligned}
& \mathcal{A}=\left[\begin{array}{lll}
1 & 0 & 0 \\
0 & \eta & 0 \\
0 & 0 & \eta
\end{array}\right], \mathcal{B}_{1}=\left[\begin{array}{ccc}
v_{1} & \eta & 0 \\
\eta & \eta v_{1} & 0 \\
0 & 0 & 0
\end{array}\right], \mathcal{B}_{2}=\left[\begin{array}{ccc}
v_{2} & 0 & \eta \\
0 & \eta v_{2} & 0 \\
\eta & 0 & \eta v_{2}
\end{array}\right], \\
& \mathcal{U}=\left[\begin{array}{c}
\eta \\
v_{1} \\
v_{2}
\end{array}\right], \mathcal{G}=\left[\begin{array}{c}
\mathcal{K}\left(\partial_{x_{1}} v_{1}+\partial_{x_{2}} v_{2}\right) \\
0 \\
0
\end{array}\right] .
\end{aligned}
$$

Multiplying (3.2) by the matrix $\mathcal{A}$ yields the following system

$$
\mathcal{A} \partial_{t} \mathcal{U}+\mathcal{B}_{1} \partial_{x_{1}} \mathcal{U}+\mathcal{B}_{2} \partial_{x_{2}} \mathcal{U}+\mathcal{G}=0
$$

Applying $J^{s}$ to (3.3) and multiplying it by $J^{s} \mathcal{U}$ show

$$
\begin{aligned}
\frac{1}{2} \frac{\mathrm{d}}{\mathrm{d} t} \int J^{s} \mathcal{U} \cdot \mathcal{A} \cdot J^{s} \mathcal{U} \mathrm{d} x & \underbrace{\frac{1}{2} \int J^{s} \mathcal{U} \cdot \partial_{t} \mathcal{A} \cdot J^{s} \mathcal{U} \mathrm{d} x}_{I I I_{1}}-\underbrace{\int J^{s} \mathcal{U} \cdot\left[J^{s}\left(\mathcal{A} \partial_{t} \mathcal{U}\right)-\mathcal{A} J^{s} \partial_{t} \mathcal{U}\right] \mathrm{d} x}_{I I I_{2}} \\
& -\underbrace{\int J^{s} \mathcal{U} \cdot\left[J^{s}\left(\mathcal{B}_{1} \partial_{x_{1}} \mathcal{U}\right)-\mathcal{B}_{1} J^{s} \partial_{x_{1}} \mathcal{U}\right] \mathrm{d} x}_{I I I_{3}}-\underbrace{\int J^{s} \mathcal{U} \cdot \mathcal{B}_{1} \cdot \partial_{x_{1}} J^{s} \mathcal{U} \mathrm{d} x}_{I I I_{4}} \\
& -\underbrace{\int J^{s} \mathcal{U} \cdot\left[J^{s}\left(\mathcal{B}_{2} \partial_{x_{2}} \mathcal{U}\right)-\mathcal{B}_{2} J^{s} \partial_{x_{2}} \mathcal{U}\right] \mathrm{d} x}_{I I I_{6}}-\underbrace{\int J^{s} \mathcal{U} \cdot \mathcal{B}_{2} \cdot \partial_{x_{2}} J^{s} \mathcal{U} \mathrm{d} x}_{I I_{7}} \\
& -\underbrace{\int}_{J^{s} \mathcal{U} \cdot J^{s} \mathcal{G} \mathrm{d} x} .
\end{aligned}
$$

Notice $s>2+a$ and $H^{1+\delta}\left(\mathbb{R}^{2}\right) \hookrightarrow L^{\infty}\left(\mathbb{R}^{2}\right)$ with $\delta \in(0, s-2-a)$. Proceed as Sect. 2.1 by using Kato-Ponce's commutator estimates or integration by parts, we then can estimate

$$
\sum_{j=1}^{6}\left|I I I_{j}\right| \lesssim\left(\|\eta\|_{H^{s}}+\|\boldsymbol{v}\|_{H^{s}}+\|\eta\|_{H^{s}}\|\boldsymbol{v}\|_{H^{s}}\right)^{3}
$$


We are left to handle the term $I I I_{7}$. Using integration by parts and the Eqs. (3.2) 2 and (3.2) 3 , we calculate

$$
\begin{aligned}
I I I_{7}= & -\int \sqrt{\mathcal{K}} J^{s} \partial_{x_{1}} \eta \cdot \sqrt{\mathcal{K}} J^{s} v_{1} \mathrm{~d} x-\int \sqrt{\mathcal{K}} J^{s} \partial_{x_{2}} \eta \cdot \sqrt{\mathcal{K}} J^{s} v_{2} \mathrm{~d} x \\
= & \int \sqrt{\mathcal{K}} J^{s}\left(\partial_{t} v_{1}+v_{1} \partial_{x_{1}} v_{1}+v_{2} \partial_{x_{2}} v_{1}\right) \cdot \sqrt{\mathcal{K}} J^{s} v_{1} \mathrm{~d} x \\
& +\int \sqrt{\mathcal{K}} J^{s}\left(\partial_{t} v_{2}+v_{2} \partial_{x_{2}} v_{2}+v_{1} \partial_{x_{1}} v_{2}\right) \cdot \sqrt{\mathcal{K}} J^{s} v_{2} \mathrm{~d} x \\
= & \frac{1}{2} \frac{\mathrm{d}}{\mathrm{d} t}\left(\left(\sqrt{\mathcal{K}} J^{s} v_{1}\right)^{2}+\left(\sqrt{\mathcal{K}} J^{s} v_{2}\right)^{2}\right) \mathrm{d} x+\underbrace{\int \sqrt{\mathcal{K}} J^{s}\left(v_{1} \partial_{x_{1}} v_{1}\right) \cdot \sqrt{\mathcal{K}} J^{s} v_{1} \mathrm{~d} x}_{I I I_{8}} \\
& +\underbrace{\int \sqrt{\mathcal{K}} J^{s}\left(v_{2} \partial_{x_{2}} v_{1}\right) \cdot \sqrt{\mathcal{K}} J^{s} v_{1} \mathrm{~d} x}_{I I I_{9}}+\underbrace{\int \sqrt{\mathcal{K}} J^{s}\left(v_{2} \partial_{x_{1}} v_{2}\right) \cdot \sqrt{\mathcal{K}} J^{s} v_{2} \mathrm{~d} x}_{I I I_{10}} \\
& +\underbrace{\int \sqrt{\mathcal{K}} J^{s}\left(v_{1} \partial_{x_{1}} v_{2}\right) \cdot \sqrt{\mathcal{K}} J^{s} v_{2} \mathrm{~d} x}_{I I I_{11}} .
\end{aligned}
$$

To estimate the term $I I I_{8}$, we commute $v_{1}$ out as:

$$
I I I_{8}=\underbrace{\int v_{1} \sqrt{\mathcal{K}} J^{s} \partial_{x_{1}} v_{1} \cdot \sqrt{\mathcal{K}} J^{s} v_{1} \mathrm{~d} x}_{I I I_{12}}+\underbrace{\int\left[\sqrt{\mathcal{K}} J^{s}, v_{1}\right] \partial_{x_{1}} v_{1} \cdot \sqrt{\mathcal{K}} J^{s} v_{1} \mathrm{~d} x}_{I I I_{13}} .
$$

Integration by parts yields

$$
\left|I I I_{12}\right| \lesssim\|\boldsymbol{v}\|_{H^{s}}\left\|v_{1}\right\|_{H^{s+\frac{a}{2}}}^{2} .
$$

Using the assumptions (1.3) and (1.4), in a similar fashion to (2.13), we obtain

$$
\left|I I I_{13}\right| \lesssim\|\boldsymbol{v}\|_{H^{s}}\left\|v_{1}\right\|_{H^{s+\frac{a}{2}}}^{2} .
$$

One can similarly treat $I I I_{9}, I I I_{10}$ and $I I I_{11}$ and finally conclude that

$$
\sum_{j=8}^{11}\left|I I I_{j}\right| \lesssim\|\boldsymbol{v}\|_{H^{s}}\left(\left\|v_{1}\right\|_{H^{s+\frac{a}{2}}}^{2}+\left\|v_{2}\right\|_{H^{s+\frac{a}{2}}}^{2} .\right.
$$

It follows from (3.4)-(3.7) that

$$
\begin{aligned}
& \frac{\mathrm{d}}{\mathrm{d} t} \int\left(\left(J^{s} \eta\right)^{2}+\eta\left(\left(J^{s} v_{1}\right)^{2}+\left(J^{s} v_{2}\right)^{2}\right)+\left(\left(\sqrt{\mathcal{K}} J^{s} v_{1}\right)^{2}+\left(\sqrt{\mathcal{K}} J^{s} v_{2}\right)^{2}\right)\right) \mathrm{d} x \\
& \quad \lesssim\left(\|\eta\|_{H^{s}}+\|\boldsymbol{v}\|_{H^{s}}+\|\eta\|_{H^{s}}\|\boldsymbol{v}\|_{H^{s}}\right)^{3}+\|\boldsymbol{v}\|_{H^{s}}\left(\left\|v_{1}\right\|_{H^{s+\frac{a}{2}}}^{2}+\left\|v_{1}\right\|_{H^{s+\frac{a}{2}}}^{2}\right)
\end{aligned}
$$

We instead consider the functional

$$
\begin{aligned}
H(\eta, \boldsymbol{v})(t)= & \int\left(\left(J^{s} \eta\right)^{2}+\eta\left(\left(J^{s} v_{1}\right)^{2}+\left(J^{s} v_{2}\right)^{2}\right)+\left(J^{s+\frac{a}{2}} v_{1}\right)^{2}\right. \\
& \left.+\left(\left(\sqrt{\mathcal{K}} J^{s} v_{1}\right)^{2}+\left(\sqrt{\mathcal{K}} J^{s} v_{2}\right)^{2}\right)\right) \mathrm{d} x
\end{aligned}
$$


and assume the solution $\eta$ satisfies the non-cavitation assumption: there exists $h_{1} \in(0,1)$ such that

$$
1+\eta(t, x) \geq h_{1}, \quad(x, t) \in \mathbb{R}^{2} \times[0, T],
$$

and the upper bound assumption: there exists $h_{2}>0$ such that

$$
\eta(t, x) \leq h_{2}, \quad(x, t) \in \mathbb{R}^{2} \times[0, T],
$$

where the time $T$ will be determined by the energy estimate below. Then, as Sect. 2.1 by using (3.8) and (3.9), one obtains

$$
H(\eta, \boldsymbol{v})(t) \lesssim_{a, h_{1}, h_{2}} H(\eta, u)(0)+\int_{0}^{t}\left(H(\eta, \boldsymbol{v})(\tau)+H(\eta, \boldsymbol{v})^{3}(\tau)\right) \mathrm{d} \tau,
$$

which together with the Grönwall's inequality implies that there exists some appropriately small positive number $T$ such that

$$
H(\eta, v)(t) \leq C\left(T, a, h_{1}, h_{2}\right) H(\eta, v)(0), \quad \forall t \in[0, T] .
$$

\subsection{Estimates for the Differences of Two Solutions}

Assume $i=1$, 2. Let $\left(\eta_{i}, \boldsymbol{v}_{i}\right)$ be the solutions of (1.2) with the initial data $\left(\eta_{i 0}, \boldsymbol{v}_{i 0}\right)$ and satisfy the non-cavitation assumption (3.8) and the upper bound assumption (3.9). Consider the evolutionary equations of the difference $\mathcal{U}_{1}-\mathcal{U}_{2}$ and proceed as Sect.2.2, we then can show the estimate

$$
\tilde{H}(t) \leq C \tilde{H}(0), \quad \forall t \in[0, T],
$$

where the functional $\tilde{H}$ is given by

$$
\begin{aligned}
\tilde{H}(t)= & \int\left(\left(\eta_{1}-\eta_{2}\right)^{2}+\eta_{1}\left(\left(v_{11}-v_{12}\right)^{2}+\left(v_{21}-v_{22}\right)^{2}\right)\right. \\
& \left.+\left(J^{\frac{a}{2}}\left(v_{11}-v_{12}\right)\right)^{2}+\left(J^{\frac{a}{2}}\left(v_{21}-v_{22}\right)\right)^{2}\right) \mathrm{d} x,
\end{aligned}
$$

and $T$ is determined in (3.10) and $C=C\left(T, a, h_{1}, h_{2}, H\left(\eta_{1}, \boldsymbol{v}_{1}\right)(0), H\left(\eta_{2}, \boldsymbol{v}_{2}\right)(0)\right)$.

Acknowledgements Open access funding provided by NTNU Norwegian University of Science and Technology (incl St. Olavs Hospital - Trondheim University Hospital). The author is grateful to the referees for their comments and suggestions that helped improve the exposition of the manuscript.

Open Access This article is licensed under a Creative Commons Attribution 4.0 International License, which permits use, sharing, adaptation, distribution and reproduction in any medium or format, as long as you give appropriate credit to the original author(s) and the source, provide a link to the Creative Commons licence, and indicate if changes were made. The images or other third party material in this article are included in the article's Creative Commons licence, unless indicated otherwise in a credit line to the material. If material is not included in the article's Creative Commons licence and your intended use is not permitted by statutory regulation or exceeds the permitted use, you will need to obtain permission directly from the copyright holder. To view a copy of this licence, visit http://creativecommons.org/licenses/by/4.0/.

\section{References}

1. Aceves-Sánchez, P., Minzoni, A.A., Panayotaros, P.: Numerical study of a nonlocal model for water-waves with variable depth. Wave Motion 50, 80-93 (2013) 
2. Bona, J.L., Smith, R.: The initial-value problem for the Korteweg-de Vries equation. Philos Trans R Soc Lond Ser A 278, 555-601 (1975)

3. Carter, J.D.: Bidirectional Whitham equations as models of waves on shallow water. Wave Motion 82, 51-61 (2018)

4. Claassen, K.M., Johnson, M.A.: Numerical bifurcation and spectral stability of wavetrains in bidirectional Whitham models. Stud Appl Math 141, 205-246 (2018)

5. Colliander, J., Keel, M., Staffilani, G., Takaoka, H., Tao, T.: Sharp global well-posedness for KdV and modified KdV on $\mathbb{R}$ and T. J Am Math Soc 16, 705-749 (2003)

6. Ehrnström, M., Johnson, M.A., Claassen, K.M.: Existence of a highest wave in a fully dispersive two-way shallow water model. Arch Ration Mech Anal 231, 1635-1673 (2019)

7. Ehrnstrom, M., Wahlén, E.: On whitham's conjecture of a highest cusped wave for a nonlocal dispersive equation. Ann. Inst. H. Poincaré Anal. Non. Linéaire 36, 1603-1637 (2019)

8. Ehrnström, M., Wang, Y.: Enhanced existence time of solutions to the fractional Korteweg-de Vries equation. SIAM J. Math. Anal. 4, 3298-3323 (2019)

9. Hunter, J.K., Ifrim, M., Tataru, D., Wong, T.K.: Long time solutions for a Burgers-Hilbert equation via a modified energy method. Proc. Am. Math. Soc. 143, 3407-3412 (2015)

10. Kalisch, H., Pilod, D.: On the local well-posedness for a full-dispersion Boussinesq system with surface tension. Proc. Am. Math. Soc. 147, 2545-2559 (2019)

11. Kato, T., Ponce, G.: Commutator estimates and the Euler and Navier-Stokes equations. Commun. Pure Appl. Math. 41, 891-907 (1988)

12. Kenig, C.E., Pilod, D.: Local well-posedness for the KdV hierarchy at high regularity. Adv. Differ. Equ. 21, 801-836 (2016)

13. Klein, C., Linares, F., Pilod, D., Saut, J.-C.: On Whitham and related equations. Stud. Appl. Math. 140, 133-177 (2018)

14. Lannes, D.: The water waves problem, vol. 188 of Mathematical Surveys and Monographs, American Mathematical Society, Providence, RI (2013). Mathematical analysis and asymptotics

15. Linares, F., Pilod, D., Saut, J.-C.: Dispersive perturbations of Burgers and hyperbolic equations I: Local theory. SIAM J. Math. Anal. 46, 1505-1537 (2014)

16. Moldabayev, D., Kalisch, H., Dutykh, D.: The Whitham equation as a model for surface water waves. Phys. D 309, 99-107 (2015)

17. Nilsson, D., Wang, Y.: Solitary wave solutions to a class of Whitham-boussinesq systems. Z. Angew. Math. Phys. 70, 70 (2018)

18. Pei, L., Wang, Y.: A note on well-posedness of bidirectional whitham equation. Appl. Math. Lett. 98, 215-223 (2019)

19. Saut, J.-C., Wang, C., Xu, L.: The Cauchy problem on large time for surface-waves-type Boussinesq systems II. SIAM J. Math. Anal. 49, 2321-2386 (2017)

20. Saut, J.-C., Xu, L.: Well-posedness on large time for a modified full dispersion system of surface waves. J. Math. Phys. 53, 115606 (2012)

21. Trillo, S., Klein, M., Clauss, G.F., Onorato, M.: Observation of dispersive shock waves developing from initial depressions in shallow water. Phys. D 333, 276-284 (2016)

Publisher's Note Springer Nature remains neutral with regard to jurisdictional claims in published maps and institutional affiliations. 